\title{
In situ measurements of a homogeneous to heterogeneous transition in the plastic response of ion-irradiated $<111>\mathrm{Ni}$ microspecimens
}

\author{
Xinyu Zhao ${ }^{1}$, Daniel J. Strickland ${ }^{1}$, Peter M. Derlet ${ }^{2}$, Mo-rigen $\mathrm{He}^{1}$, You-Jung Cheng ${ }^{1}$, \\ Jue $\mathrm{Pu}^{1,3}$, Khalid Hattar ${ }^{4}$, Daniel S. Gianola ${ }^{*}$ \\ ${ }^{1}$ Department of Materials Science and Engineering, University of Pennsylvania, \\ Philadelphia, PA 19104, USA \\ ${ }^{2}$ Condensed Matter Theory Group, Paul Scherrer Institut, CH-5232 Villigen PSI, \\ Switzerland \\ ${ }^{3}$ Department of Materials Science and Engineering, Stanford University, Stanford, CA \\ 94305, USA \\ ${ }^{4}$ Sandia National Laboratories, Albuquerque, NM 87185, USA \\ *Email: Gianola@ seas.upenn.edu
}

\begin{abstract}
We report on the use of quantitative in situ microcompression experiments in a scanning electron microscope to systematically investigate the effect of self-ion irradiation damage on the full plastic response of $<111>\mathrm{Ni}$. In addition to the well-known irradiationinduced increases in the yield and flow strengths with increasing dose, we measure substantial changes in plastic flow intermittency behavior, manifested as stress drops accompanying energy releases as the driven material transits critical states. At low irradiation doses, the magnitude of stress drops reduces relative to the unirradiated material and plastic slip proceeds on multiple slip systems, leading to quasi-homogeneous plastic flow. In contrast, highly irradiated specimens exhibit pronounced shear localization on parallel slip planes, which we ascribe to the onset of defect free channels normally seen in bulk irradiated materials. Our in situ testing system and approach allows for a quantitative study of the energy release and dynamics associated with defect free channel formation and subsequent localization. This study provides fundamental insight to the nature of interactions between mobile dislocations and irradiation-mediated and damage-dependent defect structures.
\end{abstract}

Keywords: radiation effects, stress/strain relationship, nuclear materials 


\section{Introduction}

The large radiation fluxes experienced in nuclear reactors or in space can lead to extreme environments for structural materials designed to bear load for extended periods of time. Collision cascades resulting from interactions between energetic particles and materials often lead to degradation of materials properties during use, including structural effects such as neutron-induced embrittlement, accelerated subcritical cracking, creep, helium and hydrogen mediated swelling, and stress corrosion cracking [1-4]. These issues are guiding the development of new materials robust to these effects, yet adequate solutions providing long-term reliability and safety are still scarce.

Central to the deleterious effects of radiation damage are the nucleation and evolution of irradiation-mediated material defects. Highly energetic particles incident on materials displace atoms from their original lattice positions many times, which leads to an excess of defects in the form of self-interstitial atoms and vacancies. The unit of damage given by displacement per atom (dpa) is commonly employed to describe the extent of interaction between energetic particles and the target material. In face-centered cubic materials (fcc), a number of post-irradiation transmission electron microscopy (TEM) experiments [5-7] and atomistic simulations [8-10] have shown that atomic displacement cascades resulting from neutron, proton, and ion fluxes and subsequent creation of excess vacancies and self-interstitial atoms lead to two common microstructural features at room temperature: stacking fault tetrahedra (SFT) and dislocation loops [11]. Whereas high stacking fault energy materials (e.g. Pd) show a predominance of sessile self-interstitial Frank loops [6], low stacking fault energy materials such as $\mathrm{Cu}$ show a larger fraction of SFT $[12,13]$. One macroscopic manifestation of this new population of material defects resulting from irradiation is strengthening and hardening [1,2]. In some irradiationinduced strengthening models, both defect classes are considered to serve as dispersed barriers to dislocation motion and source operation resulting in increasing yield strength 
with defect density (and thus irradiation dose), in analogy to classic Fleischer solid solution strengthening [14]. Other models, such as the cascade-induced source hardening model ascribe the increase in yield strength to the creation of glissile dislocation loops that undergo thermally activated motion towards dislocations, subsequently pinning them in place [15]. Molecular dynamics (MD) and discrete dislocation (DD) simulations have also shown dislocation pinning by irradiation-induced defect clusters, absorption of defects by dislocations, and driven dislocation cross-slip $[9,16]$. In experimental work on irradiated fcc and bcc materials, the superposition of strengthening components arising from dislocation pinning at defect clusters [15,17] and dispersed barriers [18] was shown to describe measurements of both yield points and flow stresses reasonably well [6].

Apart from the well documented strengthening and embrittlement that occurs owing to radiation damage, fcc metals and alloys exhibit a strong tendency for plastic localization in discrete bands oriented along shear directions, leading to unstable and unpredictable mechanical behavior $[19,20]$. These bands, which have characteristic spacings of the order of $100 \mathrm{~nm}$, are often called dislocation- or defect free channels and were first observed in bec metals [21,22]. Diaz de la Rubia et al. used a multiscale modeling approach to show defect-free channel formation in $\mathrm{Cu}$ and $\mathrm{Pd}$ by progressive cross-slip and double-cross-slip mechanisms involving interactions between moving dislocations and irradiation-induced defects [23]. DD simulations performed by Khraishi et al. showed SFT in $\mathrm{Pd}$ and $\mathrm{Cu}$ to be weaker pinning points compared with sessile Frank dislocation loops, whereas unfaulting of Frank loops and absorption by mobile dislocations led to defect-free channel formation [24]. Arsenlis and colleagues showed via MD simulations of bcc-Fe that a critical density of irradiation-induced dislocation loops was necessary to transition from homogeneous to heterogeneous plastic deformation, with the latter regime governed by defect-free channel creation and subsequent highly localized slip [25]. In situ TEM studies have also provided substantial 
insight to the nature of interactions between mobile dislocations and sessile defects [2630]. In situ straining of irradiated stainless steel demonstrated locking at irradiationinduced defects of previously mobile dislocations [28]. Dislocation sources were still active during this mode, suggesting propagation, not nucleation, to be the controlling factor. Despite these atomistic insights, the previously described analytical models $[14,15]$ and experiments $[6,12]$ cannot quantitatively link the onset or characteristics of these plastic localization events with applied stress and defect density. Notably, the nature of crystalline slip before and after channel formation, the magnitude of energy release during a plastic instability, and a quantitative measure of the slip statistics during plastic flow across a wide range of irradiation doses have yet to be experimentally provided. Such information is crucial input for informing predictive models and validating atomistic simulations that are dependent on quasi-empirical interatomic potentials.

The use of ion and proton irradiation has been widely used as a surrogate for neutron irradiation and captures many of the effects shown in neutron studies, including inter- and intragranular defect microstructure and chemistry, radiation hardening, and stress corrosion cracking behavior [31,32]. Small scale mechanical testing is ideally suited for systematic investigations of ion irradiation-mediated mechanical behavior, since ion penetration depths are often in the nano- and micrometer range. Miniaturized testing thus avoids complex interpretation of results obtained from irradiated bulk samples, which inevitably display gradients in defect densities across specimen dimensions [33-35]. Moreover, quantitative in situ approaches enable direct correlations between measured response and plastic instabilities governed by dislocation radiation-induced defect interactions. Kiener et al. recently reviewed the application of small scale methods to the testing of irradiated metals and showed that experiments producing nominally uniaxial states (in contrast to e.g. nanoindentation) were ideal, particularly in the presence of 
inhomogeneous irradiation conditions [34]. Despite progress in the understanding of irradiation-induced defect and property correlations, no studies have systematically elucidated the dose dependence of plastic behavior or analyzed the statistics of plastic intermittency across the critical dose for defect free channel formation.

In this paper, we apply novel small scale in situ mechanical testing approaches to self-ion irradiated $<111>\mathrm{Ni}$ to systematically investigate damage-dependent defect property correlations. In addition to radiation-induced strengthening, our study focuses on the transition from homogeneous to heterogeneous plastic flow across over two orders of magnitude of dose and the nature of intermittency in crystalline slip in the presence of dispersed obstacles. We study the evolution of defect-induced microstructure with increasing damage and correlate this structure with plasticity and the propensity for shear localization. The statistics of slip provide insight to the key defect interactions in the presence of obstacles of varying density and size. The objective of this study is to make experimental connections to atomistic simulation and theory, and ultimately pave the way for designing and understanding more complex materials and microstructures with enhanced radiation tolerance, such as nanocrystals, multilayers, multi-phase nanostructured alloys.

\section{Experiments and Materials}

\subsection{Material preparation and ion irradiation experiments}

The pillars were milled out of $6.4 \mathrm{~mm}$ diameter $99.995+\%$ nickel $<111>$ single crystal that was mechanically polished to a mirror finish prior to ion implantation. The high purity $\mathrm{Ni}$ was implanted with $35 \mathrm{MeV} \mathrm{Ni}^{6+}$ using a $6 \mathrm{MV}$ Tandem accelerator at Sandia's Ion Beam Lab. The samples were irradiated with a nearly parallel dc beam of approximately $0.15 \mathrm{~mm}^{2}$. The unsuppressed current was measured on a floating sample holder and calibrated to a Faraday cup directly upstream from the sample. The full 
profiles of irradiation damage distributions (Fig. 1a) were calculated using a Monte-Carlo based stopping range of ions in matter (SRIM) modeling program [36], which provides the end of range and dpa values in each irradiation region as given in Table 1. We note that a small gradient in dose is expected from the sample surface to the base of the pillar; thus, we report the average dose $\langle\beta>$ in each region by integrating the dose profile within the specimen gage section.

\subsection{Micro-pillar fabrication}

Cylindrical microcompression specimens were machined into the surface of each irradiated region by using top down annular milling $[37,38]$ with a focused $\mathrm{Ga}^{+}$ion beam (FEI Strata DB 235 dual-beam focused ion beam and scanning electron microscope). A cascaded milling procedure with progressively decreasing ion currents (final polishing step using $50 \mathrm{pA}$ ) was employed to fabricate the cylindrical specimens using an ion beam voltage of $30 \mathrm{kV}$. Annular pedestals were included in the geometry to provide clear imaging for in situ mechanical testing. During the final milling step, multiple iterations with decreasing pattern size were used to minimize the taper of the pillar and result in smooth specimen sidewalls. The nominal geometry of the pillars with diameters of $1 \mu \mathrm{m}$ and heights of $3 \mu \mathrm{m}$ was selected based on several considerations, including $35 \mathrm{MeV} \mathrm{Ni}^{6+}$ end of range $(4.39 \pm 0.41 \mu \mathrm{m})$ and an optimal pillar aspect ratio (2 to 3$)$ to minimize the effects of buckling [39]. This puts the end of range of ions well into the base beneath the microspecimen, as illustrated in Fig. 1a. Moreover, we chose a constant pillar diameter for our study to avoid convolution of irradiation effects with intrinsic size effects. We note that a transition to bulk-like behavior in proton-irradiated $\mathrm{Cu}$ micropillars was reported to occur at pillar diameters of approximately $0.5 \mu \mathrm{m}$, above which the strengthcontrolling length scale was attributed to the irradiation-induced defect microstructure, not the pillar diameter [34]. While this size transition is presumably dependent on both the material (e.g. stacking fault energy, vacancy diffusion coefficients) and the irradiation 
conditions [33,34], we expect our measurements of $\mathrm{Ni}$ specimens to be reasonable indicators of bulk behavior, particular since our dpa levels are much higher than in Ref. [33]. At least 3 specimens from each of the 4 irradiated and unirradiated regions were prepared following the procedure above. Pillar dimensions at various locations along the specimen length were measured from high-resolution SEM images.

A representative as-fabricated pillar is shown in Fig 1b. The pillars exhibited a small taper angle of $3.2 \pm 0.7^{\circ}$. Specimen diameters measured from the mid-plane of the pillar $\left(<d_{\text {mid }}>=1.17 \pm 0.15 \mu \mathrm{m}\right)$ were used to calculate engineering stresses. All fabricated pillars exhibited heights smaller than the irradiation damage range $(<h>=2.70 \pm 0.40$ $\mu \mathrm{m})$, resulting in pillar aspect ratios within the ideal range of 2 to 3 . We note that a gradient in the damage profile is expected along the compression axis of the pillar; our calculations show the mean gradient within the gage section to be approximately $0.01 \mathrm{dpa}$ $\mathrm{nm}^{-1}$, with the gradient being the smallest near the punch-pillar interface where plasticity is expected to commence. In addition, any near surface irradiation effects from $30 \mathrm{kV} \mathrm{Ga}{ }^{+}$ milling of pillars is expected to be negligible compared to the damage introduced from 35 $\mathrm{MeV} \mathrm{Ni}^{+}$irradiation.

\subsection{Microstructural characterization}

To characterize the microstructure of irradiated samples and quantify the damagedependent defect density of the material, TEM lamellas were carefully prepared and liftout using a focused ion beam. A protective Pt layer was deposited prior to the milling process to reduce the defects induced by the $\mathrm{Ga}+$ ion penetration. Lamellas were milled using an ion beam with progressively decreasing ion currents at a voltage of $30 \mathrm{kV}$, which is followed by a cleaning process using an ion beam voltage of $5 \mathrm{kV}$ to remove preparation artifacts. The thicknesses at various locations of lamellas were measured from high-resolution scanning electron microscope (SEM) images to convert 
measurements of defect densities to corresponding volumetric densities.

Defect microstructures of both as-prepared and ion-irradiated samples were examined using a JEOL 2100 TEM operated at $200 \mathrm{kV}$. Dark-field images of cross-sectional specimens were carried out in approximately the $\mathbf{g}(3 \mathbf{g})$ weak-beam mode, with the

reciprocal space vector $\mathbf{g}$ selected as $(200)^{*}$ for most samples, where the * denotes the reciprocal space vector based on the indexed diffraction patterns. Quantification of defect microstructures was performed in regions of all lamella approximately $1 \mu \mathrm{m}$ below the surface.

\subsection{In situ SEM compression}

Quantitative in situ mechanical testing of irradiated and unirradiated microcompression specimens was performed using a custom-built micro- and nanomechanical testing platform [40-42] installed in a high resolution SEM (FEI Quanta field-emission environmental SEM). We took advantage of the large volume available in the vacuum chamber (often not possible in more confined imaging systems such as TEM) to integrate a fully instrumented testing, manipulation, and alignment platform. The small-scale testing system consists of three primary components: (1) a stiff piezoelectric actuator operated in closed-loop control mode with an onboard strain gauge sensor $(1 \mathrm{~nm}$ resolution), which enables direct displacement-controlled testing, (2) a 6 degree-offreedom (3 orthogonal translational and 3 orthogonal rotational) closed-loop nanopositioning system (SmarAct SmarPod, with $1 \mathrm{~nm}$ and $1 \mu$ rad resolution), and (3) a capacitive based force sensing probe (Femtotools FT-S10000 Microforce Sensing Probe, with $0.5 \mu \mathrm{N}$ resolution at $10 \mathrm{~Hz}$ acquisition rate). The testing apparatus as mounted in the SEM chamber is shown in Figs. $2 \mathrm{a}$ and $2 \mathrm{~b}$. A square flat punch with a diameter of approximately $12 \mu \mathrm{m}$ was milled via FIB at the tip of the silicon probe of the load cell for compression testing. 
Special considerations were made in the design of the in situ testing system to eliminate the effects of misalignment between the flat punch (and thus the load cell axis) and the testing specimen, thus ensuring uniaxial loading. Optimal alignment is achieved primarily through the use of the closed-loop nanopositioning stage. The in-plane ( $x-y$ plane in Fig. 2) alignment adjustment is relatively straightforward and achieved by rotation $\left(\theta_{z}\right)$ and translation of the tip relative to the specimen with feedback based on SEM observation. More challenging is the out-of-plane alignment ( $y-z$ plane), as shown in Fig. 2c We achieve the optimal alignment by maximizing the contact stiffness as a function of rotation angle $\theta_{x}$ during low load compression experiments, as illustrated in Fig. 2c. Data for one such optimization procedure are shown (Fig. 2c) to approximately describe a cosine function, consistent with simple geometry. These experiments are first performed on the surface area adjacent to the pillar and subsequently on the pillar itself to refine the alignment. Real-time load-displacement measurements are acquired to ensure elastic loading of the specimen.

Pillar compression tests were operated in displacement control to achieve nominal strain rates of approximately $10^{-3} \mathrm{~s}^{-1}$ and SEM images were obtained concurrently during testing with the fast scan direction of the beam rastering oriented parallel to the pillar compression axis to minimize convolution of imaging and testing time scales.

\section{Results}

\subsection{Microstructural and Damage Characterization}

Representative weak-beam dark-field TEM images of the cross-sectional specimens in the near-surface region of as-prepared and ion-irradiated samples at different damage levels $(0.25,2.5,25$, and $40 \mathrm{dpa})$ are shown in Figs. 3(a-e). As a standard process, the [011] zone axis (see the inset of Fig. 3(a)) was obtained first, then the specimen was 
slightly tilted so that a reflection $\mathbf{g}=(200)^{*}$ was strongly excited. The spot $\mathbf{g}$ was finally moved onto the optic axis by tilting the electron beam, thus realizing an approximate $\mathbf{g}(3 \mathbf{g})$ weak-beam condition, which was then used for central dark-field imaging. As Fig. 3(a) shows, the interior of the as-prepared sample showed very weak contrast associated with damage induced by the FIB preparation process under such imaging conditions, which thus provided a good reference for determination of defect densities due to controlled self-ion irradiation.

Figs. 3(b-e) show the general microstructure of Ni specimens irradiated to a damage level of $0.25,2.5,25$ and 40 dpa respectively. A large number of fine defect structures $\left(\sim 10^{23}\right.$ $\mathrm{m}^{-3}$ ) were produced after ion irradiation, presumably a combination of dislocation loops and SFTs as has been previously reported $[4,13]$. There is no clear evidence of aligned defect clusters or defect cluster wall formation in any of the specimens. The number density of defect clusters in each region of the current study was computed using image processing and analysis, wherein connected components in thresholded and binarized images are identified as defect clusters and their areal densities computed. The apparent sizes of defect clusters are, however, very dependent on the specific diffraction conditions and precluded direct comparison across all irradiated samples. Results of the defect density analysis is shown as a function of damage level in Fig. 3(f), plotted alongside literature reports of defect density measurements of irradiated $\mathrm{Ni}(<1 \mathrm{dpa})[4,13]$. Our data at the low end of dpa agree well with literature, show a slight increase in defects density with increasing damage level, and reach an apparent saturation level at higher damage ( 2.5 dpa). For low damage levels $(\leq 2.5 \mathrm{dpa})$, the distribution of defect sizes as well as their spatial distribution within the crystal is relatively uniform. However, higher damage ( $>2.5 \mathrm{dpa}$ ) results in evidence of defect coarsening in addition to heterogeneity in their spatial positioning, thereby changing the nature of the dispersed obstacle "forest." 


\subsection{Mechanical Response and Plastic Deformation Morphology}

Compressive stress-strain curves of specimens subjected to different displacement damage levels $(0.25,2.5,25$, and $40 \mathrm{dpa})$ in comparison with unirradiated pillars are shown in Fig. 4. Beginning with the unirradiated pillars (i.e. 0 dpa, Fig. 4a), we measure an apparently linear elastic regime in the initial loading stages, followed by yielding at stresses of approximately $400 \mathrm{MPa}$. Such a proportionality limit was measured in the majority of specimens characterized, which was used for defining the yield stresses. The ensuing plastic flow response is characterized by intermittency, manifested as sudden stress drops followed by periods of re-loading to subsequent critical states, which ultimately defines a serrated flow behavior over large plastic strain values. Such a response is the hallmark of single crystalline slip controlled by dislocation avalanches as observed in many fcc micro- and nanoscale specimens tested at room temperature [4346], with the important distinction that most reported experimental stress-strain curves demonstrate strain bursts (not stress drops), owing to the load-controlled nature of the testing systems. Some degree of hardening, as defined by the increase of the timeaveraged flow stress compared with the macroscopic yield stress, is detected in the unirradiated pillars, which qualitatively agrees with other experiments on $<111>\mathrm{Ni}$ pillars of similar sizes, suggesting dislocation-dislocation interactions or dislocation pileup in our pillars oriented for multiple slip with low Schmid factors $(m=0.27)$ [45]. Overall, our results for the unirradiated pillars quantitatively agree, within the scatter of the measured strengths, with the study of $<111>$ Ni by Frick et al. [45].

Microcompression pillars fabricated from irradiated regions exhibited distinct and damage-dependent mechanical behavior. At low damage levels (0.25 and 2.5 dpa, Figs. $4 \mathrm{~b}$ and $4 \mathrm{c}$ ), an increase in both yield and flow stresses is measured, with higher doses resulting in higher strengths. In addition, intermittency is still evident in the plastic flow regime. Interestingly, the 0.25 dpa specimens show some net hardening similar to the 
unirradiated pillars, whereas the 2.5 dpa specimens exhibit softening at large total strains. Most notably, the amplitude of the serrations in the stress-strain curves during the plastic flow regime decrease with increasing dose, with the 2.5 dpa specimens showing relatively smooth flow behavior over intermediate strain scales. Upon plastic evolution, a gradual softening occurs, with a reduction of approximately $15 \%$ of the peak flow strength at $15 \%$ strain. Notably, the scale of the load drop drops becomes much finer at 2.5 dpa, suggesting relatively homogeneous plastic flow response owing to the suppression of large dislocation avalanche events. Importantly, we note that the smaller magnitude load drops are still measurable above the instrumental noise floor, which we characterized independently.

Whereas increasing ion irradiation dose to 25 and 40 dpa results in progressively higher yield strengths, with the 40 dpa specimens exhibiting yield strengths as high as $880 \mathrm{MPa}$ ( $>2$ times the unirradiated yield strength), we observe a large change in the plastic flow response. Heterogeneous behavior emerges with the occurrence of pronounced stress drops followed by small amplitude intermittency at progressively increasing plastic strain. In two of the 25 dpa specimens, a large stress drop (as large as $80 \mathrm{MPa}$ ) is detected at or near the first sign of macroscopic yielding. Subsequent stress drops exhibit mean amplitudes similar to those of the lower dose samples. Despite the occurrence of these large slip events, further flow behavior appears to be stabilized against softening, albeit at stresses lower than the peak stress. One of the 25 dpa specimens (Fig. 4d) exhibited a second large stress drop at approximately $10 \%$ strain with a duration extending over $~ 2 \%$ strain. In some instances, the occurrence of the large slip events and corresponding slip offset changed the load bearing capability of the specimen and presumably the stress state upon further loading, as manifest in distinct unloading slopes (e.g. red curve in Fig. 4d). In contrast to the $2.5 \mathrm{dpa}$ specimens, significant scatter in the response is measured from pillar to pillar at high dpa. We note that the apparent hardening measured in one of the 40 
dpa specimens (black curve in Fig. 4e) is attributed to contact between the slipped region of the pillar and surrounding material in the pedestal, which was observed in situ.

The apparent transition from heterogeneous flow behavior in the unirradiated pillars to homogeneous response at low irradiation dose $(0.25$ and $2.5 \mathrm{dpa})$, followed by a distinct heterogeneous behavior at high dose (25 and $40 \mathrm{dpa}$ ), is further corroborated by postmortem SEM images of deformed microcompression pillars as shown in Fig. 5. A representative unirradiated pillar, unloaded at a small strain of $\sim 5 \%$, shows characteristic slip offsets at the pillars surfaces consistent with the orientation of multiple $\{111\}$ slip planes available for plastic flow with equivalent Schmid factors, as has been reported for this loading orientation [45]. With the introduction of damage from low dose ion irradiation, slip steps at the surface become vanishingly small, as shown for representative 0.25 (Fig. 5b) and 2.5 dpa (Fig. 5c) specimens. In contrast, the 25 (Fig. 5d) and 40 dpa (Fig. 5e) pillars exhibit singular and pronounced slip steps, along with additional slip traces with smaller offsets on parallel planes. Taken as a whole, whereas jerky slip occurs on multiple planes for unirradiated pillars, the transition to heterogeneous plastic deformation at high irradiation doses is characterized by the occurrence of highly localized shear event(s) occurring on parallel $\{111\}$ planes. We also detect some spatial extent (i.e. thickness) of the large shear localization events normal to the slip plane, as noted by the slightly jagged nature of the surface step (e.g. Fig. 4e). A 40 dpa pillar cross-sectioned using the FIB through a shear localization feature shows the magnitude of the shear offset to be approximately $80 \mathrm{~nm}$, which corresponds to roughly $300<110>$-type dislocations.

Our in situ testing approach allows us to directly correlate plastic morphology with features measured in the stress-strain response, as shown in the image sequence and corresponding stress-strain curve for a 40 dpa specimen in Fig. 6. Following linear 
elastic loading, the nucleation of a shear localized event correlates with macroscopic yielding of the specimen (Fig. 6f). Interestingly, the nucleation of this shear band (Fig. 6b) was observed on the cylinder surface within the gage section of the specimen, rather than at the pillar/punch interface, as is commonly seen in micro- and nanocompression experiments due to friction or stress concentrations at this interface [38]. This is presumably due to the small gradient of dose along the length of the pillar, with the largest extent of damage concentrated near the base of the pillar. As defect-free channel nucleation in irradiated metals generally occurs at stress concentrations [26], this suggests that the stress owing to dislocation-defect interactions is higher than any amplification of stress at the pillar-punch interface. Subsequent propagation of the shear band proceeds until it intersects with the top surface of the pillar (Fig. 6c). Most notably, full propagation of this single shear band event correlates with a measured stress drop (denoted as point $\mathrm{c}$ in Fig. 6f). Subsequent intermediate slip events on parallel planes are associated with smaller magnitudes of stress drops (e.g. Fig. 6d). This combination of measurement and direct observation suggests that the large load drops are associated with large localization events at high dose, which we associate with the onset of dislocation free channels as discussed in subsequent sections.

\section{Discussion}

\subsection{Irradiation-Induced Strengthening}

Our results show a marked increase in both yield and flow strengths with increasing irradiation dose over the studied range, with the yield strength approximately doubling at a dose of $40 \mathrm{dpa}$, as shown in Fig. 7. The trend of strength vs. dose shows apparent saturation response with increasing dpa. This observation is qualitatively consistent with post-mortem $[13,47]$ and in situ [48] characterization of damage microstructure in other fcc metals, where a sub-linear defect density evolution with increasing dose and eventual saturation has been reported. This behavior is attributed to distinct evolution stages 
characterized by defect incubation, ultimately followed by defect interactions that lead to decreasing defect generation rates that increase with dose [48]. However, we note that no such saturation behavior has been reported for $\mathrm{Ni}$ for doses as high as $1 \mathrm{dpa}[4,13]$, which is consistent with our yield strength results.

The increases in yield strength measured in our irradiated Ni pillars can be compared with predictions from the widely used dispersed barrier hardening model [14], which gives:

$$
\Delta \sigma_{Y}=\alpha M \mu b \sqrt{\rho_{d} d_{\text {defect }}},
$$

where $\alpha$ reflects the obstacle strength ( $\alpha=0.2$ for weak obstacles), $M$ is the Taylor factor (3.1 for $<111>$ axial compression), $\mu$ is the shear modulus ( $76 \mathrm{GPa}$ for $\mathrm{Ni}$ ), $b$ is the Burgers vector magnitude $(0.249 \mathrm{~nm}$ for fcc $\mathrm{Ni}), \rho_{d}$ is the mean defect density, and $d_{\text {defect }}$ is the mean size of the defects. Here we can see the strengthening is not only influenced by the defect density, but also by the defect size. By analyzing the dependence of strengthening on $\rho_{d}$ and the known constants for our material, we can examine the influence of the defect size and heterogeneity on our measured response. Applying Eq. 1 directly to our experimental measurement of mean yield strength in Fig. 7 and defect density in Fig. 3(f) and assuming a constant defect size (i.e. a denser defect population with fixed size) yields $d_{\text {defect }}$ of approximately $2 \mathrm{~nm}$. Despite this being a reasonable value that falls between reported values of SFT and dislocation loop sizes in irradiated $\mathrm{Ni}$ [13], the overall fit to our data (not shown) is rather poor. This is reasonably explained by a defect population which coarsens with increasing dpa, as has been shown in radiation damage studies of Ni [13] albeit at smaller dpa. Indeed, by assuming that $d_{\text {defect }}$ varies with dpa in Eq. 1 we find a best fit to our strengthening data that indicates a defect size that nearly doubles between 2.5 and $25 \mathrm{dpa}$, consistent with our TEM observations. We note that this empirical treatment ignores polydispersity in the defect distributions and changes in the obstacle strength (via $\alpha$ ), which presumably affects the propensity for 
shear localization. Analyzing the full plastic deformation response in the flow regime and quantifying the associated avalanche behavior can provide further insight to the nature of dislocation irradiation-induced microstructure interactions.

\subsection{Plastic Response in the Flow Regime}

We now examine correlations between the measured plastic response and the irradiationinduced microstructure. The unirradiated mechanical response (0 dpa), shown in Fig. 4a, reflects the typical behavior of a single crystalline micro-deformation experiment $[43,45,49]$ : discrete plasticity and stochasticity resulting in a strong scatter in plastic response. The former reflects the intermittency of dislocation network evolution and the latter that the sample volume is not of a sufficient size to self-average. Thus, from the perspective of a bulk metal, the Weibull modulus representing yield or flow strength is presumably low.

The 0.25 dpa deformation curves exhibit a significant reduction in scatter allowing for a reproducible plastic flow regime and also a finer distribution of stress drops, which quite generally suggests the introduction of an internal length scale which suppresses intermittent plasticity at the larger strain scales. However, this irradiation-induced microstructure is evidently not resistant enough to strongly affect the yield and flow stresses. These points suggest the introduction of a well-defined density of defects which act as weak pinning sites for the 0.25 dpa sample, which is consistent with our TEM observations shown in Fig. 2(b). That the density is well defined across different samples is demonstrated by the reduction in scatter in the overall flow response, which would be reflected by a higher Weibull modulus and thus better self-averaging.

The 2.5 dpa deformation curves exhibit a similar scatter as the 0.25 dpa samples and also a strengthening. The fact that there is a slightly narrower distribution of stress drops 
suggests a slightly higher density of defects; again consistent with our TEM measurements. This increase in defect density can give rise to the observed strengthening as described by the dispersed barrier hardening model via an increase in $\rho_{d}$ of Eq. 1 . However, the measured softening with evolving plastic strain suggests that the initial defect structure is not stable against an applied stress and/or plastic deformation. Nevertheless, as the scatter in response does not increase with plastic strain, this does not suggest a coarsening of the defect density with plastic evolution, but rather a relaxation of stronger defect sites introduced during irradiation. Thus, upon irradiation to $2.5 \mathrm{dpa}$, the defect density has increased compared to the 0.25 dpa sample, but the more important effect is that the defects themselves have generally become stronger dislocation obstacles (via an increase of $\alpha$ or the defect size in Eq. 1). We note that a change in the ratio of dislocation loops to SFTs would elicit a similar effect. Notably, this evolution represents a change in defect structure which appears to not be stable against deformation.

For the more damaged 25 dpa and 40 dpa specimens, the significant increase in scatter and the strengthening suggests a dilute density of relatively strong defects coexisting with a finer defect density (of a nature seen in the low dosage samples). This heterogeneity in the irradiation-induced defect distribution is qualitatively supported by our microstructural observations. The dilute density of strong defects is evidently reasonably stable against deformation and, owing to the scatter, must have a mean separation that is not that much smaller than the sample size; the conditions for self averaging are clearly not met which is reflected by the measured scatter in the compressive response.

The above mechanistic scenarios generally assume that the differences in plastic evolution are a result of differences in the initial defect structure. Thus, apart from the softening seen in the 2.5 dpa sample, a static picture is proposed and shown schematically in Fig. 8. The motivation for taking a static rather than a dynamic viewpoint is based on 
the view that micro-pillar deformation experiments are considered to generally operate in a microplastic regime, where structural evolution and hardening is generally considered to be absent. Indeed, several reports have shown through quantitative measurements of microstructure evolution during straining that the dislocation density does not change dramatically in the flow regime [50-52]. Moreover recent work has shown that the avalanche velocities occurring during intermittent plasticity in micro-compression tests are largely independent of the applied stress, suggesting a plasticity that is not associated with macroscopic flow but rather with that of micro-plasticity where the pre-existing internal stresses dominate [53].

The transition to a heterogeneous flow response at high damage levels ( $25 \mathrm{dpa}$ and higher) and the occurrence of shear localization within thin bands are consistent with observations of defect-free channels, which are thought to result from the annihilation of irradiation-induced defects owing to dislocation-defect reactions [10,16,23,26,29,30,54]. Gliding dislocations may interact with SFT or sessile loops, although the annihilation reactions require high stresses and small defect spacings [54], which explain the transition at some critical damage level. However, the creation of a channel free from defect debris would likely promote further slip localization in these regions, which would result in continued plastic flow characterized by large load drops of the order of the unirradiated sample. Our results, rather, show relatively small amplitudes of flow intermittency following large load drops (with a scale comparable to low dpa samples) and in some instances further hardening, which suggest that the fine distribution of irradiation-induced defects governs the majority of plastic flow even after shear localization occurs. This difference, together with the fact that only small numbers of plastic events occur during loading, point towards the observation of the onset of the defect free channel phenomenon rather than that of a fully developed defect free channel that is seen in ex situ analysis of bulk samples which have been heavily irradiated. While 
further detailed microstructural characterization is needed to confirm this hypothesis, our results on the whole point to the importance of a non-uniform population of irradiationinduced defects in defining the transition from homogeneous to heterogeneous plastic flow.

\subsection{Statistical Analysis of Stress Drops}

The qualitative trend of decreasing amplitude of plastic flow serrations, as measured from the intermittent response of the mechanical response, with increasing dose in the intermediate damage regime $(<5 \mathrm{dpa})$ suggests that mobile dislocations encounter an increasingly dense field of obstacles on their slip planes, thus reducing the energy of each avalanche. This behavior is followed by the occurrence of mesoscopic shear localization at high doses (25 and $40 \mathrm{dpa}$ ), resulting in discrete and relatively large stress drops accompanying these slip events. Interestingly, the magnitudes of flow serrations following large localization events are still smaller than those from the unirradiated specimens. Thus, examining the full statistics of stress drop events as a function of irradiation dose could provide insight into the nature of the mobile dislocation and irradiation-mediated defect interactions.

To analyze the serrated nature of stress-strain curves, we employed a frequency domain analysis of the load signals measured during in situ compression testing. We first measured the frequency response of our instrument by directly compressing the force sensor with our piezoelectric actuator, and subsequently decoupled the instrument response from that of the material in the frequency domain. To eliminate these and other noise components, we applied a low-pass Butterworth filter with a cutoff frequency of 1.2 $\mathrm{Hz}$ to the load-time signal. The selection of the cutoff frequency is informed by the power spectrum measured during direct compression of the force sensor. The filtered signal is transformed back to the time domain and individual avalanches are extracted 
(see Figure 9). The minimum experimentally measurable avalanche magnitude is limited by the noise of the system, which is found to vary from sample to sample likely owing to small differences in the boundary conditions of the pillars. To quantify the noise level, we perform a linear fit to the filtered load-time response during unloading, where we expect avalanche activity to be negligible. The RMS error between the linear fit and the signal is a measure of the system noise and we set a minimum avalanche magnitude threshold of twice the RMS error.

Fig. 10 shows the complementary cumulative distribution functions of stress drops measured from our specimens as a function of irradiation state. The population of load drop events tends to shift to smaller magnitudes for irradiated samples, increasingly so with increasing dose. The minimum event sizes measured in irradiated samples with varying dose are comparable, but substantially lower than that of the unirradiated sample ( 20\%), suggesting the influence of irradiation-induced obstacles in controlling the smallest avalanches. We note that the 2.5 dpa samples were found to have a noise level $\sim 50 \%$ larger than the noise level found in the other samples. Therefore, the number of small avalanches found in the 2.5 dpa samples is less than the number found in the other samples. The evolution of the maximum stress drop is non-monotonic with dose, with the maximum drops in the $25 \mathrm{dpa}(221 \mathrm{MPa})$ and $40 \mathrm{dpa}$ (108 $\mathrm{MPa})$ samples comparable to the unirradiated sample (149 $\mathrm{MPa})$ and the maximum drops in the $0.25 \mathrm{dpa}(56.6 \mathrm{MPa})$ and $2.5 \mathrm{dpa}(38.0 \mathrm{MPa})$ significantly smaller. The largest avalanches corresponded to system spanning shear localization events, as observed during in situ deformation, suggesting the onset of defect-free channels. However, the large fraction of relatively small load drops following the large avalanches (smaller than the unirradiated sample) suggests that a portion of the defect population persists and controls subsequent avalanches. In samples with intermediate damage ( $<25 \mathrm{dpa})$, the mean spacing between irradiation-induced defects is sufficiently small so as to control the largest possible 
avalanches, and evidently stable with continuing plastic strain.

To quantify these observations on stress drop statistics, we fit a power-law with an exponential cutoff of the form:

$$
P(X \geq x) \propto x^{-\tau+1} \exp \left[-\left(\frac{x}{x_{o}}\right)^{2}\right],
$$

to the complementary cumulative distribution functions (CCDF) of stress drops for each irradiation state in the range $0.10<P \leq 1.00$ (see Fig. 11). The exponent $\tau$ describes the power-law behavior of $P$ in the scale-free size regime and the cutoff magnitude $x_{o}$ quantifies the stress-drop magnitude at which $P$ begins to deviate from power-law behavior. The cutoff value in stress reflects the presence of length scales that are either dynamical, such as a dislocation correlation length, or static, such as a sessile microstructural length scale. Values of $\tau$ and $x_{o}$ for each irradiation level are shown in Table 2.

The magnitude of the scaling exponent $\tau \approx 1.5$ is similar to that found in experiments [44,55] and simulations [46] of micrometer-sized crystals. The major effect of irradiation on the stress drops statistics is in the change of $x_{o}$ with irradiation dose. The four-fold decrease in $x_{o}$ in the 0.25 dpa samples as compared to the unirradiated samples is the result of a significant population of obstacles to dislocation motion. These obstacles, which arrest dislocation motion, impose an upper bound on the magnitude of stress drops to which power-law scaling holds. Further irradiation damage increases $x_{o}$, indicative of a slight increase in the spacing of obstacles. As discussed previously, strong obstacles develop at higher doses of irradiation (25 and $40 \mathrm{dpa}$ ). The presence of these strong obstacles is reflected in the significant excess in the experimental CCDF above $P$ found 
at stress drop magnitudes above $x_{o}$ (see Fig. 11, d and e), which support the hypothesis of a polydisperse and heterogeneous population of defect clusters at high dose.

In summary, the data of Figs. 10 and 11 show that for the low damage (0.25 and $2.5 \mathrm{dpa}$ ) samples, the distribution of stress drops is more strongly truncated when compared to the distribution of the unirradiated material. For the high damage (25 and 40 dpa) samples, the distributions additionally exhibit strong tails that cannot be accounted for by eqn. 2 . This latter aspect is most probably due to the relatively few large-stress-drop events occurring due to the low number of strong/large defect sites within each pillar (see Figs. $8 \mathrm{~d}$ and $8 \mathrm{e}$ for a schematic realization) resulting in poor self-averaging and a behavior that varies from sample to sample.

For the lower stress-drop scale, where eqn. 2 represents reasonably well the data of Figs. 10 and 11 for all dosages, the exponential scaling function indicates the emergence of a upper cut-off stress scale upon irradiation. Assuming that the statistics of stress-drops are equivalent to plastic strain burst magnitudes [56], this also indicates the emergence of an upper plastic strain cutoff scale.

The above trends can be rationalized by modern theories of scale free plasticity, in which the dislocation network is considered to be partially (or fully) in a self-organized critical state [57]. Currently there are two main variants of the universality class associated with the plastic deformation of crystalline materials, those for which the system is in a state of criticality irrespective of the applied external stress $[46,58,59]$ and those for which the system is only in a state of criticality at (or close to) a critical depinning stress [60,61].

Considering the first case, then finite system scaling enters directly into the scaling functions and the plastic strain cutoff scale is controlled in a simple way by a 
characteristic length scale over which a dislocation structure can propagate. Such a viewpoint is compatible with the creation, upon irradiation, of an upper length scale due to a population of pinning defects as envisaged in the schematic pictures of Fig. 8. In addition, the very large stress drops (plastic strain magnitudes) could well be due to a larger upper length scale induced by a dilute population of stronger pinning sites. However for this latter part, caution should be exercised due to the already mentioned poor statistics.

For the second universality class, in which the plastic strain scaling is dominated by a correlation length scale now set by how close the applied stress is to the critical depinning stress, truncation becomes a measure of how close the system is to being critical. With respect to this picture, the stronger truncation seen in the irradiated samples suggests, upon irradiation, the effective critical depinning stress increases taking the material further away from criticality for the applied stress values of Fig. 4. If however, a micro-structural/finite-system-size cutoff length scale exists which is comparable to or less than the depinning correlation length then scaling is controlled in a similar way to the first universality class. Work by Freidman et al. [62] suggests micro-deformation experiments agree with the mean field depinning picture in which a transition to true finite size scaling occurs only in the sub $500 \mathrm{~nm}$ pillar diameter regime. This result implies that a population of radiation induced defects, whose mean free spacing is well below $500 \mathrm{~nm}$, would dominate the truncation of the plastic strain magnitude distribution in a similar way as that of the first universality class.

\section{Conclusions}

In summary, our results on, and analysis of, the microcompression of self-ion irradiated $<111>$ Ni pillars subjected to displacement damage levels as high as $40 \mathrm{dpa}$ form the basis for the following conclusions: 
- Irradiation-induced strengthening was measured in both yield and flow strengths and was consistent with the development of damage microstructures with increasing defect density that we measured in TEM. However, the dispersed barrier hardening model only captures the measured behavior when considering an evolution of either the obstacle strength or the defect size with increasing dpa, with the latter supported by TEM observations. This suggests the development of not only a finer mean defect separation, but also a heterogeneous defect population as dpa is increased.

- Upon deformation, all pillars exhibited stochastic behavior and intermittent plasticity, both of which were affected by the extent of irradiation-induced damage.

- When compared to the unirradiated specimens, the deformation properties of the low dpa (0.25 and $2.5 \mathrm{dpa})$ specimens showed reduced scatter, from specimen to specimen, and therefore a more reproducible mechanical response. In addition, the decreasing magnitude of flow serrations and onset of softening at large strains in the $2.5 \mathrm{dpa}$ specimens point to a finer and stronger defect microstructure. These features allow for sufficient self-averaging of plastic events within the finite size of the pillar thereby reducing the scatter and magnitude of load drops during flow. However, the measured softening suggests that the microstructure is not particularly stable at large plastic strains.

- At large dpa (25 and $40 \mathrm{dpa}$ ), a striking heterogeneity emerges in the compressive response and deformation morphology, wherein strengthening competes with large stress drops corresponding to system-spanning dislocation avalanches. The occurrence of both large and fine scale serrations in the compressive response, as well as stochastic response from specimen to specimen, implies the coexistence of irradiation-induced defect populations with distinct strengths and characteristic length scales. The onset of such a microstructure evidently is responsible for the 
transition from homogeneous to heterogeneous plastic flow at a dpa level somewhere between 2.5 and 25 dpa.

- Statistical analysis of the load drop magnitudes as a function of dpa are consistent with theories of scale free plasticity, and exhibit power law behavior with exponents of $\tau \approx 1.5$ that are approximately invariant with dpa. However, the values of the finite size cutoff $x_{\mathrm{o}}$ are affected by the irradiation-induced defect microstructure, with a four-fold reduction in $x_{\mathrm{o}}$ of the 0.25 dpa relative to the unirradiated specimens. Furthermore, $x_{\mathrm{o}}$ is non-monotonic with dpa, consistent with the development of a heterogeneous defect population. The strong heterogeneity in plastic response at high dpa (25 and $40 \mathrm{dpa}$ ), as well as the occurrence of several large stress drops that are observed as large shearing events during in situ testing, are reflected in the strong tails of the distribution at large stress drop magnitude. The trends measured in the statistics of stress drops can be ascribed to dislocation networks, brought about by the ion-induced displacement damage and strongly influenced by the damage level, that are to some degree in a self-organized critical state.

This study as a whole provides new fundamental insight to the nature of interactions between mobile dislocations and irradiation-mediated and damage-dependent defect structures in single crystalline fcc metals, providing a framework for rational integration and design of materials in applications prone to extreme radiation fluxes such as nuclear fission and fusion reactors. Designing next-generation materials based on irradiationinduced strengthening and hardening considerations alone would overlook the dramatic changes in plastic flow that can occur and thereby the nature of failure.

\section{Acknowledgements}


This research was supported in part by an NSF CAREER Award \#DMR-1056293. The authors thank the support of the staff and facilities at the Nanoscale Characterization Facility at the University of Pennsylvania. K. Hattar and D.S. Gianola also acknowledge the Division of Materials Science and Engineering, Office of Basic Energy Sciences, U.S. Department of Energy. Sandia National Laboratories is a multi-program laboratory managed and operated by Sandia Corporation, a wholly owned subsidiary of Lockheed Martin Corporation, for the U.S. Department of Energy's National Nuclear Security Administration under contract DE-AC04-94AL85000.

\section{References}

[1] Was GS. Fundamentals of Radiation Materials Science: Metals and Alloys. 2007.

[2] Zinkle S, Was G. Acta Mater 2013;61:735.

[3] Zinkle S. Fusion Eng Des 2005;74:31.

[4] Zinkle SJ. Comprehensive Nuclear Materials. Elsevier; 2012.

[5] Zinkle SJ, Snead LL. J Nucl Mater 1995;225:123.

[6] Victoria M, Baluc N, Bailat C, Dai Y. J Nucl Mater 2000;276:114.

[7] Zinkle SJ, Singh BN. J Nucl Mater 2006;351:269.

[8] Kadoyoshi T, Kaburaki H, Shimizu F, Kimizuka H, Jitsukawa S, Li J. Acta Mater 2007;55:3073.

[9] Marian J, Martínez E, Lee H-J, Wirth BD. J Mater Res 2009;24:3628.

[10] Bacon DJ, Gao F, Osetsky YN. J Nucl Mater 2000;276:1.

[11] Zinkle SJ, Matsukawa Y. J Nucl Mater 2004;329-333:88.

[12] Zinkle SJ, Horsewell A, Singh BN, Sommer WF. J Nucl Mater 1994;212-215:132.

[13] Hashimoto N, Byun TS, Farrell K. J Nucl Mater 2006;351:295.

[14] Fleischer R. Acta Metall 1963;11:203. 
[15] Singh BN, Foreman AJE, Trinkaus H. J Nucl Mater 1997;249:103.

[16] Bacon DJ, Osetsky YN, Rong Z. Philos Mag 2006;86:3921.

[17] Trinkaus H, Singh BN, Foreman AJE. J Nucl Mater 1997;251:172.

[18] Fleischer RL. J Appl Phys 1962;33:3504.

[19] Singh BN, Zinkle SJ. J Nucl Mater 1993;206:212.

[20] Dai Y, Victoria M. Acta Mater 1997;45:3495.

[21] Eyre BL. Philos Mag 1962;7:2107.

[22] Tucker RP, Wechsler MS, Ohr SM. J Appl Phys 1969;40:400.

[23] Rubia T de la, Zbib H, Khraishi T. Nature 2000;489.

[24] Khraishi T, Zbib H. Mater Trans B 2002;33.

[25] Arsenlis A, Rhee M, Hommes G, Cook R, Marian J. Acta Mater 2012;60:3748.

[26] Robach JS, Robertson IM, Wirth BD, Arsenlis A. Philos Mag 2003;83:955.

[27] Suzuki M, Fujimura A, Sato A. Philos Mag 1991:37.

[28] Briceño M, Fenske J, Dadfarnia M, Sofronis P, Robertson IM. J Nucl Mater 2011;409:18.

[29] Briceño M, Kacher J, Robertson IM. J Nucl Mater 2013;433:390.

[30] Kacher J, Liu G, Robertson I. Micron 2012;43:1099.

[31] Mansur L. J Nucl Mater 1994;216:97.

[32] Was G, Busby J, Allen T, Kenik E. J Nucl Mater 2002;300:198.

[33] Kiener D, Hosemann P, Maloy SA, Minor AM. Nat Mater 2011;10:608.

[34] Kiener D, Minor AM, Anderoglu O, Wang Y, Maloy SA, Hosemann P. J Mater Res 2012;27:2724.

[35] Sharon JA, Hattar K, Boyce BL, Brewer LN. Mater Res Lett 2013;00:1. 
[36] Ziegler JF, Ziegler MD, Biersack JP. Nucl Instrum Meth Phys Res B 2010;268:1818.

[37] Volkert CA, Minor AM. MRS Bull 2007;32:389.

[38] Uchic M, Dimiduk D. Mater Sci Eng A 2005;400-401:268.

[39] Zhang H, Schuster B, Wei Q, Ramesh K. Scr Mater 2006;54:181.

[40] Magagnosc DJ, Ehrbar R, Kumar G, He MR, Schroers J, Gianola DS. Sci Rep 2013;3:16.

[41] Magagnosc DJ, Kumar G, Schroers J, Felfer P, Cairney JM, Gianola DS. Acta Mater 2014;74:165.

[42] Brugarolas T, Gianola DS, Zhang L, Campbell GM, Bassani JL, Feng G, Lee D. ACS Appl Mater Interfaces 2014;6:11558.

[43] Dimiduk DM, Uchic MD, Parthasarathy TA. Acta Mater 2005;53:4065.

[44] Dimiduk DM, Woodward C, Lesar R, Uchic MD. Science 2006;312:1188.

[45] Frick C, Clark B, Orso S. Mater Sci Eng A 2008;489:319.

[46] Csikor FF, Motz C, Weygand D, Zaiser M, Zapperi S. Science 2007;318:251.

[47] Yu K, Bufford D, Sun C, Liu Y, Wang H. Nat Comm 2013;4:1377.

[48] Li N, Hattar K, Misra a. J Nucl Mater 2013;439:185.

[49] Uchic MD, Dimiduk DM, Florando JN, Nix WD. Science 2004;305:986.

[50] Maaß R, Uchic MD. Acta Mater 2012;60:1027.

[51] Norfleet DM, Dimiduk DM, Polasik SJ, Uchic MD, Mills MJ. Acta Mater 2008;56:2988.

[52] Oh SH, Legros M, Kiener D, Dehm G. Nat Mater 2009;8:95.

[53] Maaß R, Derlet PM, Greer JR. Small 2014:1.

[54] Taylor P, Osetsky YN, Rodney D, Bacon DJ. 2008:37. 
[55] Dimiduk DM, Nadgorny EM, Woodward C, Uchic MD, Shade PA. Philos Mag 2010;90:3621.

[56] Maaß R, Wraith M, Uhl JT, Greer JR, Dahmen KA. Unpublished work.

[57] Zaiser M. Adv Phys 2006;55:185.

[58] Zaiser M, Nikitas N. J Stat Mech Theory Exp 2007;2007:P04013.

[59] Ispánovity PD, Laurson L, Zaiser M, Groma I, Zapperi S, Alava MJ. Phys Rev Lett 2014;112:235501.

[60] Dahmen K, Ben-Zion Y, Uhl J. Phys Rev Lett 2009;102:175501.

[61] Derlet PM, Maaß R. Model Simul Mater Sci Eng 2013;21:035007.

[62] Friedman N, Jennings AT, Tsekenis G, Kim J-Y, Tao M, Uhl JT, Greer JR, Dahmen KA. Phys Rev Lett 2012;109:095507. 


\section{Figure Captions}

Figure 1: (a) Schematic showing microcompression pillar specimen geometry with the pillar axis oriented parallel to the direction of $\mathrm{Ni}^{+}$ion irradiation. Dose profiles for irradiated regions $\langle\boldsymbol{\beta}\rangle=\mathbf{0 . 2 5}$ dpa (blue curve), $\langle\beta>=2.5$ dpa (green curve), $\langle\beta>=25$ dpa (orange curve), and $\langle\beta>=40$ dpa (white curve) are superimposed showing the variation of dose throughout the pillar and pillar base. Note that the peak dose is within the pedestal, with a relatively weak gradient of dose throughout the pillar gage section. (b) Tilted SEM view of a representative FIB-fabricated pillar and its pedestal.

Figure 2: (a) Image of custom in situ micro- and nanomechanical testing apparatus installed in a field-emission SEM. (b) Low-magnification SEM image showing force sensor with fabricated $12 \mu \mathrm{m} x$ $12 \mu \mathrm{m}$ flat punch at tip in close proximity to microspecimens. Coordinate system shows translational $(x, y, z)$ and rotational $\left(\theta_{x}, \theta_{y}, \theta_{z}\right)$ degrees-of-freedom for alignment. (c) Method of out-of-plane alignment using contact stiffness optimization procedure, wherein small strain elastic loading is performed to measure contact stiffness as a function of misalignment angle $\theta_{x}$. The maximum contact stiffness represents the optimal alignment.

Figure 3: Weak beam DF-TEM micrographs showing microstructure of (a) unirradiated and irradiated regions of the <111> Ni sample for doses of (b) 0.25 , (c) 2.5, (d) 25, and (e) $40 \mathrm{dpa}$. (f) Quantification of defect density as a function of damage level in comparison to literature values for single crystalline Ni [13]. The orientation of the $g$ vector in image (c) is unknown.

Figure 4: Compressive engineering stress vs. engineering strain curves for microspecimens fabricated in (a) unirradiated and irradiated regions of the <111> Ni sample for doses of (b) 0.25 , (c) 2.5, (d) 25, and (e) 40 dpa. The colors correspond to the responses of distinct specimens subjected to the same irradiation conditions.

Figure 5: SEM images of post-compressed pillars from (a) unirradiated and irradiated regions of the $<111>\mathrm{Ni}$ sample for doses of (b) 0.25, (c) 2.5, (d) 25, and (e) $40 \mathrm{dpa}$. (f) Longitudinal cross-section of compressed pillar from 40 dpa region highlighting large shear band following localization event. The image in (d) corresponds to the red curve in Fig. 4d, where the different unloading slope is related to the large slip offset resulting in the partial loss of contact between the flat punch and pillar.

Figure 6: In situ SEM testing sequence (a-d) of 40 dpa <111> Ni pillar exhibiting shear localization event. In (b) nucleation of a slip event is detected at the pillar surface within the gage section (indicated by arrow), which subsequently propagates (c) until it intersects the pillar top surface. (d) Multiple slip events occur on parallel planes, as shown in (e) tilted view. (f) The corresponding stress strain curve obtained for this experiment highlights stress drops associated with shear localization. 
Figure 7: Dependence of compressive yield and flow strengths (at $8 \%$ total strain) on $\mathrm{Ni}^{+}$irradiation dose. The closed symbols denote the current study, whereas the open symbols show comparison between unirradiated pillars and literature reports of <111> and <269> Ni pillars with similar diameters $[43,45]$. Inset shows measured data on semi-log scale, indicating a saturation of strengthening with increasing damage.

Figure 8: Schematic of proposed characteristics of irradiation-induced defect microstructure as a function of damage (b-e). At low dpa ( 0.25 and 2.5 dpa), defect clusters are fine and of uniform size and spatial distributions. At high dpa (>2.5 dpa), clusters coarsen and exhibit size polydispersity and variations in spatial positioning, leading to heterogeneous plastic response.

Figure 9: Filtered (blue) and unfiltered (black) load-time data for a (a) 0 dpa specimen, (b) 2.5 dpa specimen, and (c) 40 dpa specimen. Green circles indicates the start of a stress drop and the subsequent red circles indicates the end of the drop. The minimum stress drop magnitude is determined by analyzing the noise in the signal during unloading. Note the different scales of the axes.

Figure 10: Statistics of stress drops determined by the analysis of load-time response (see text for details). Complementary cumulative probability distribution of stress drops with data plotted on loglog scale for unirradiated and irradiated pillars of varying dose.

Figure 11: Fitting to the complementary cumulative distribution functions for the (a) unirradiated, (b) 0.25 dpa, (c) $2.5 \mathrm{dpa}$, (d) $25 \mathrm{dpa}$, and (e) 40 dpa samples. 


\section{(a)}

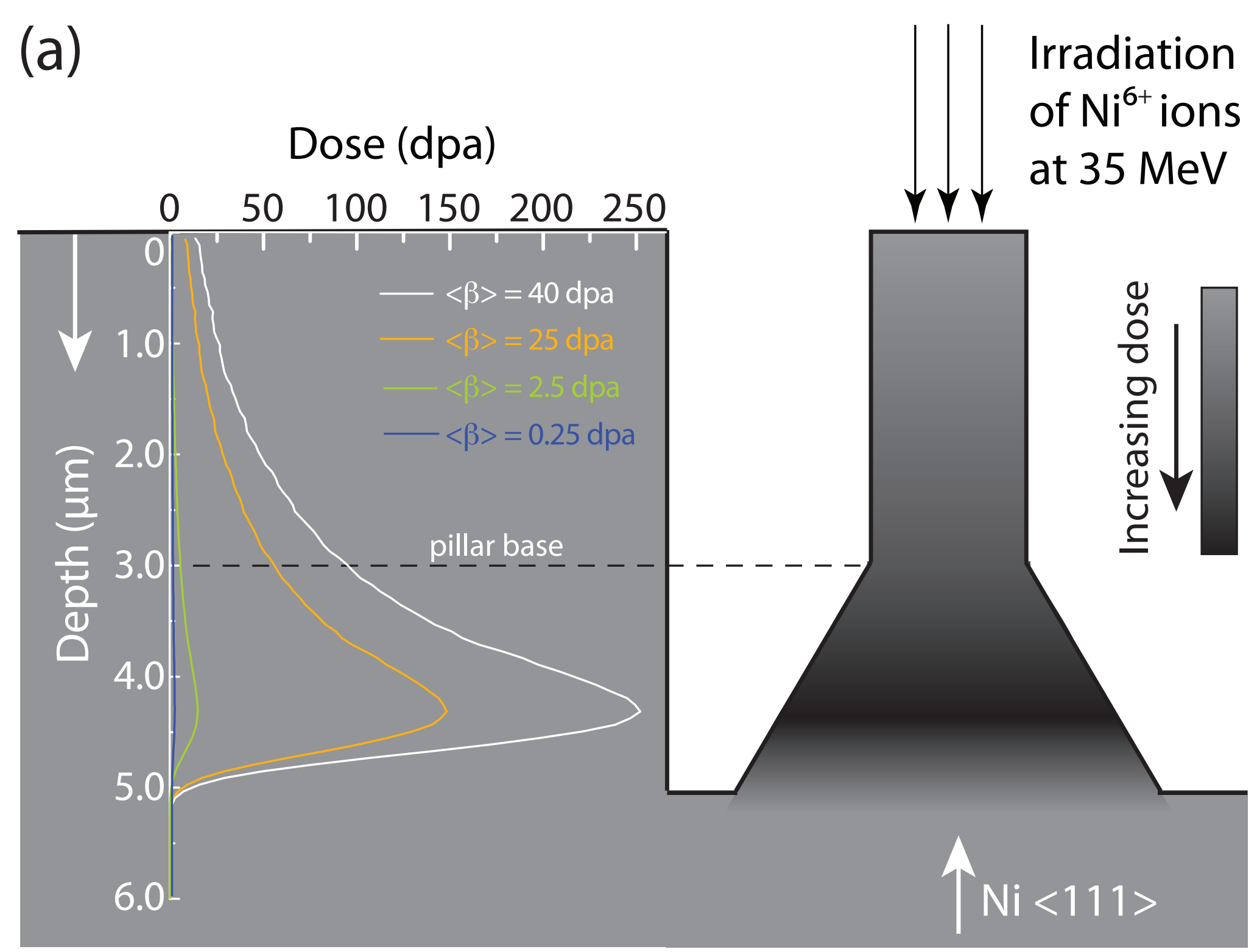

$\mathrm{Ni}<111>$
Dose (dpa) $(b)$

Irradiation of $\mathrm{Ni}^{6+}$ ions at $35 \mathrm{MeV}$

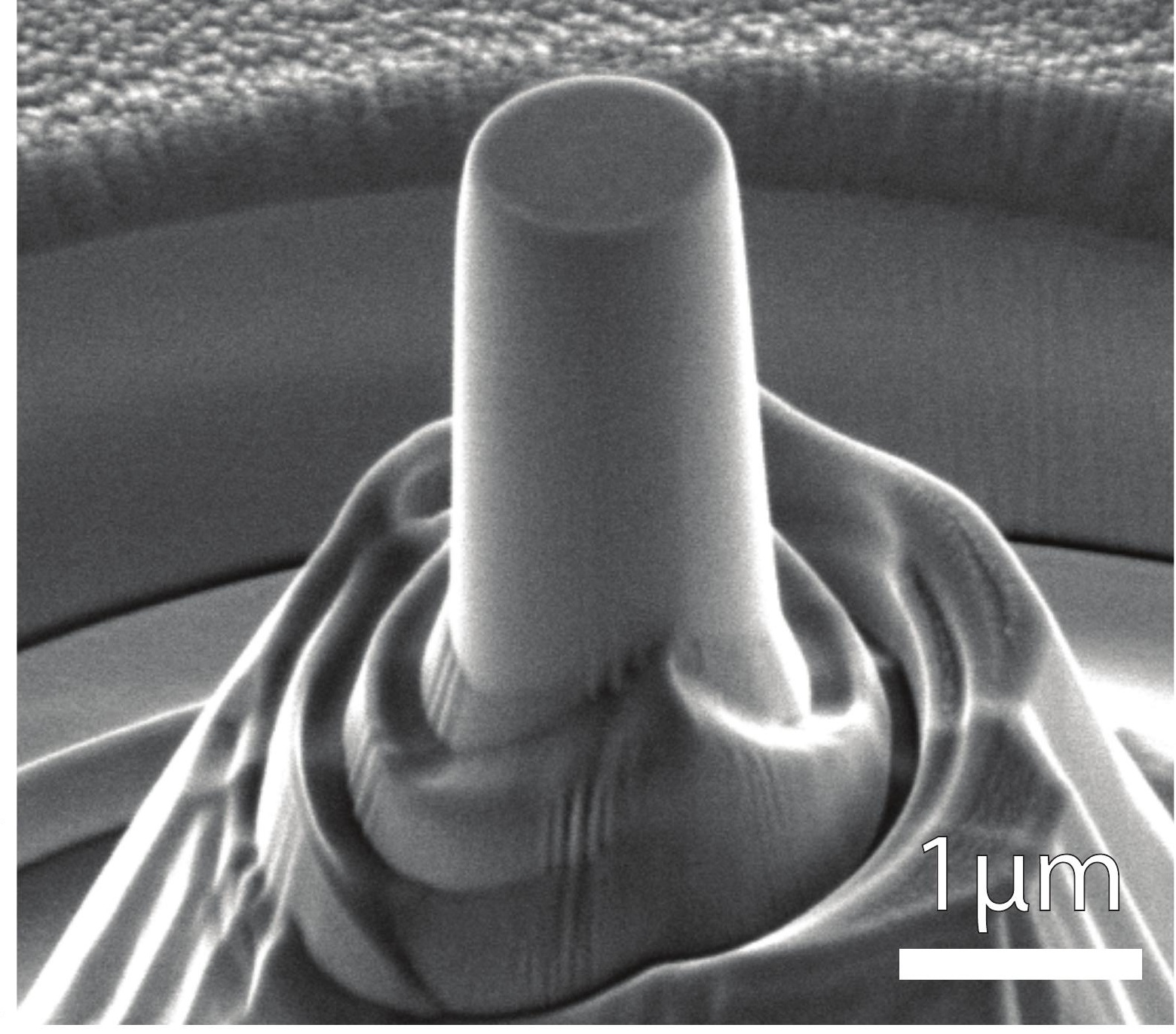

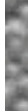




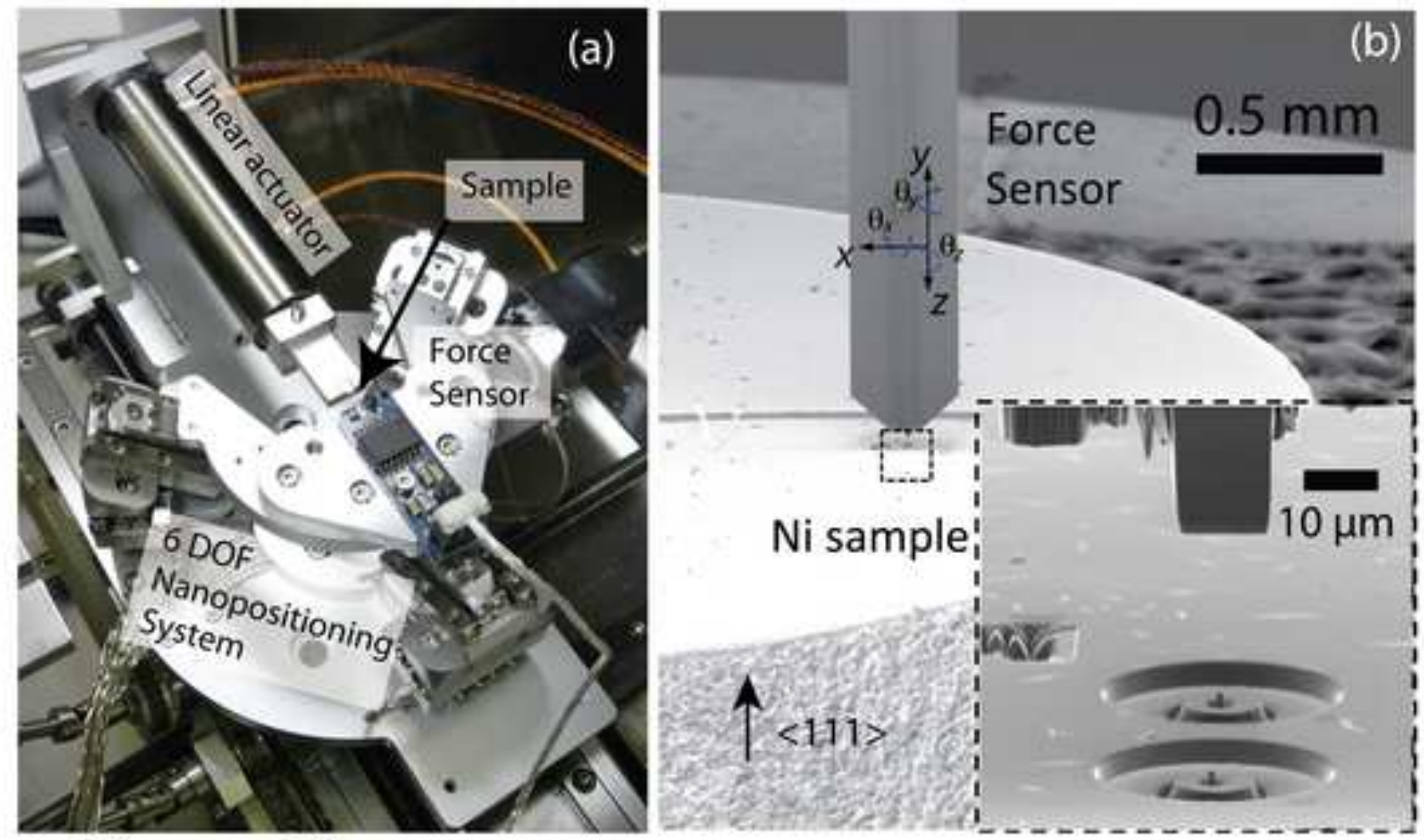

(c)

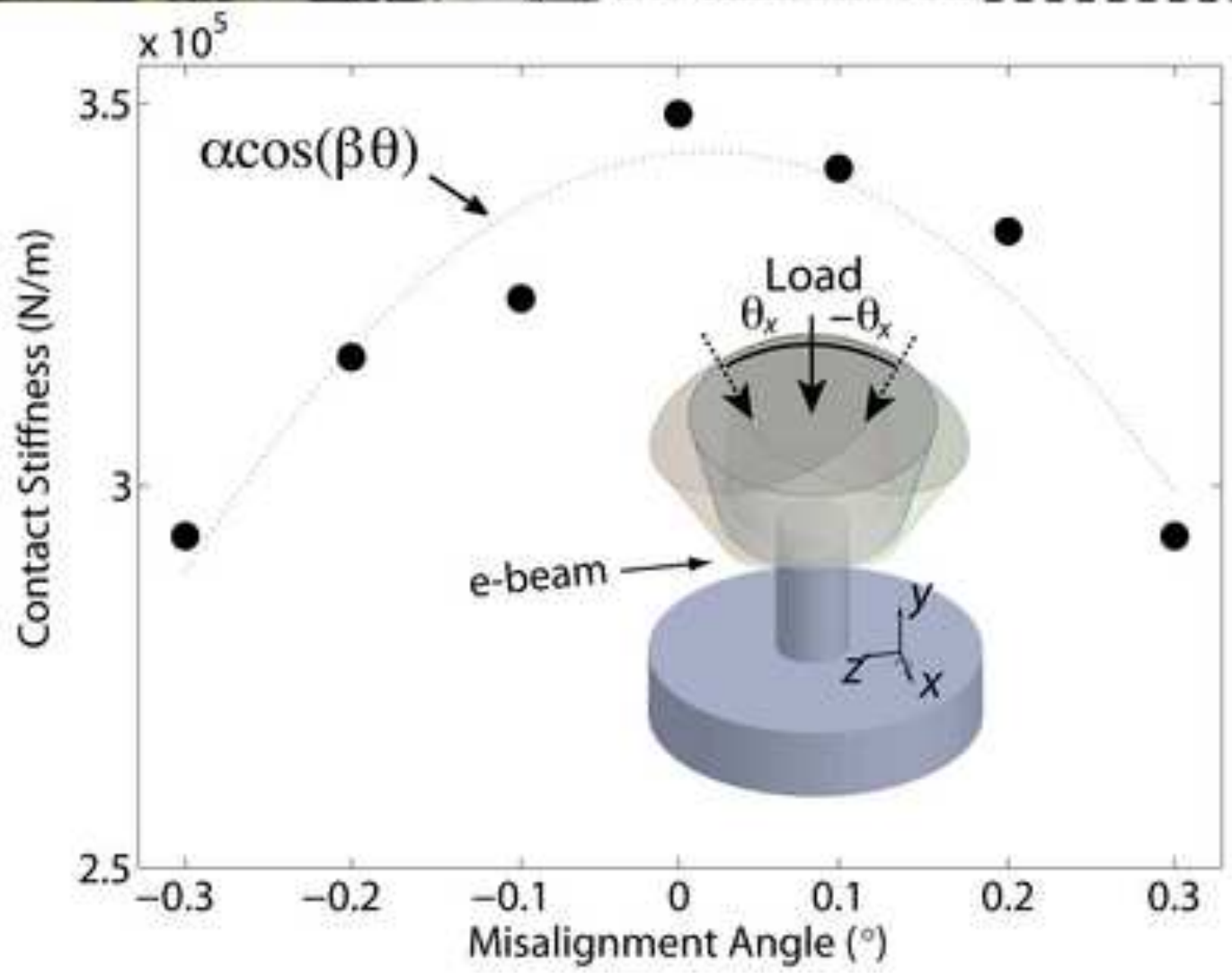



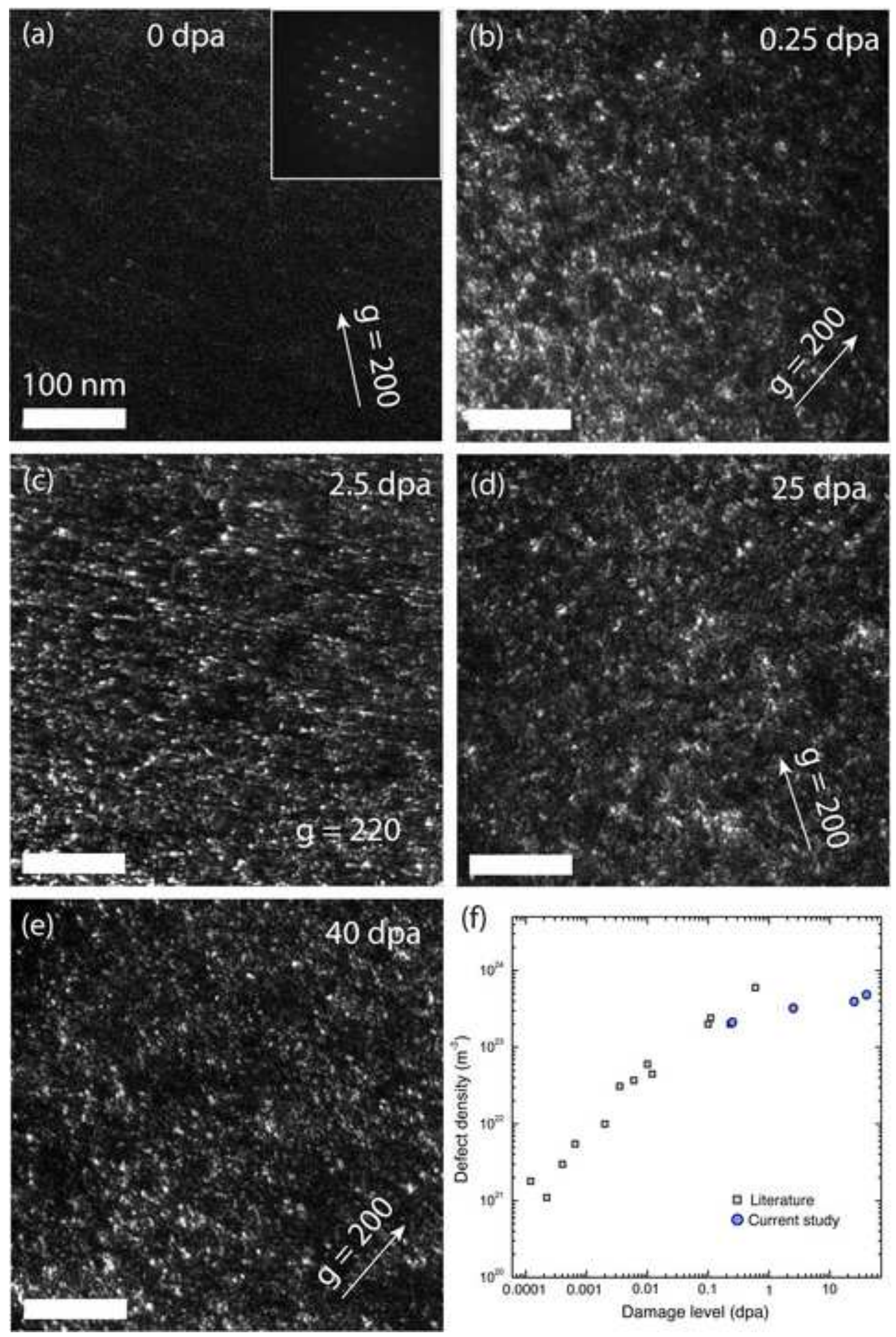

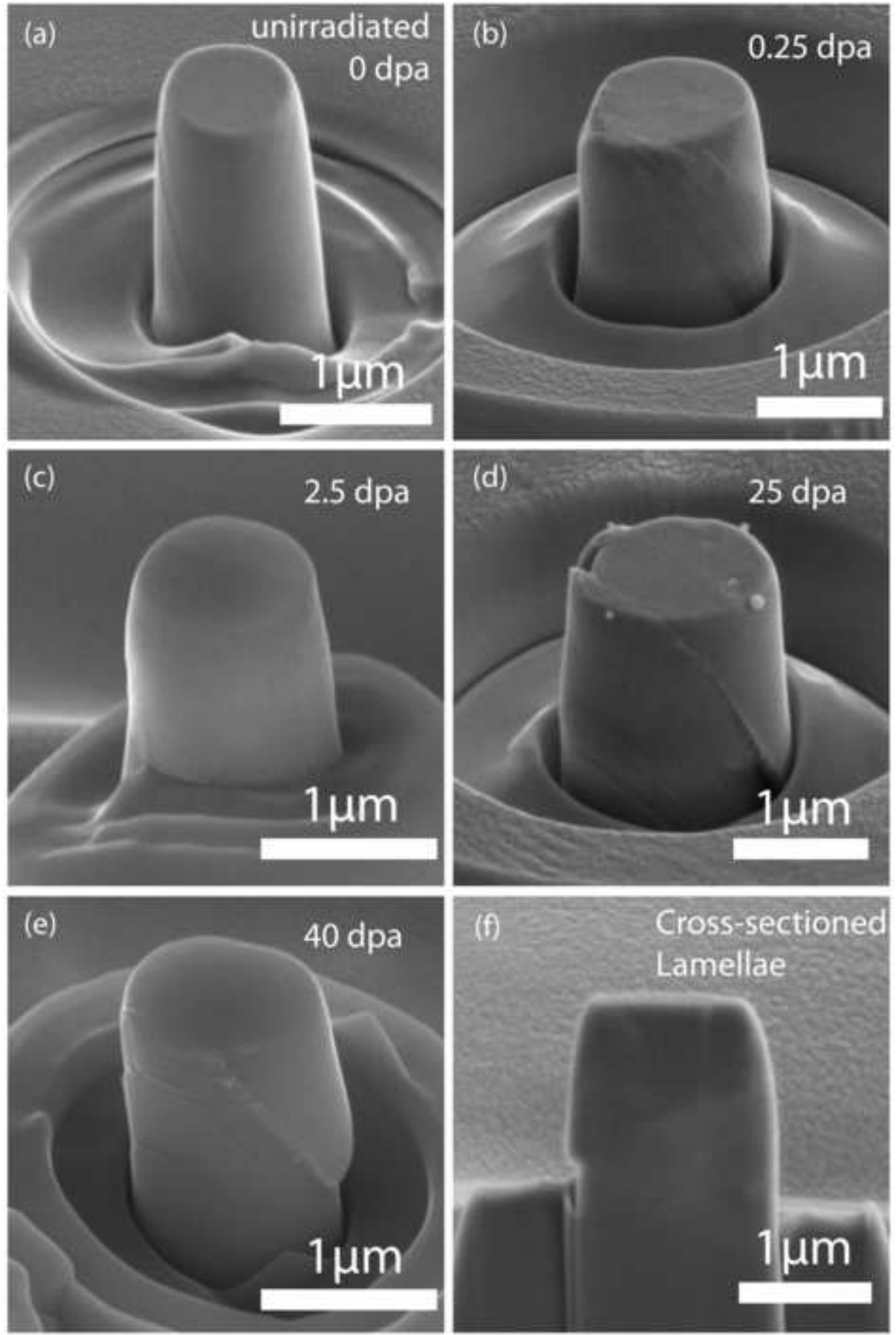

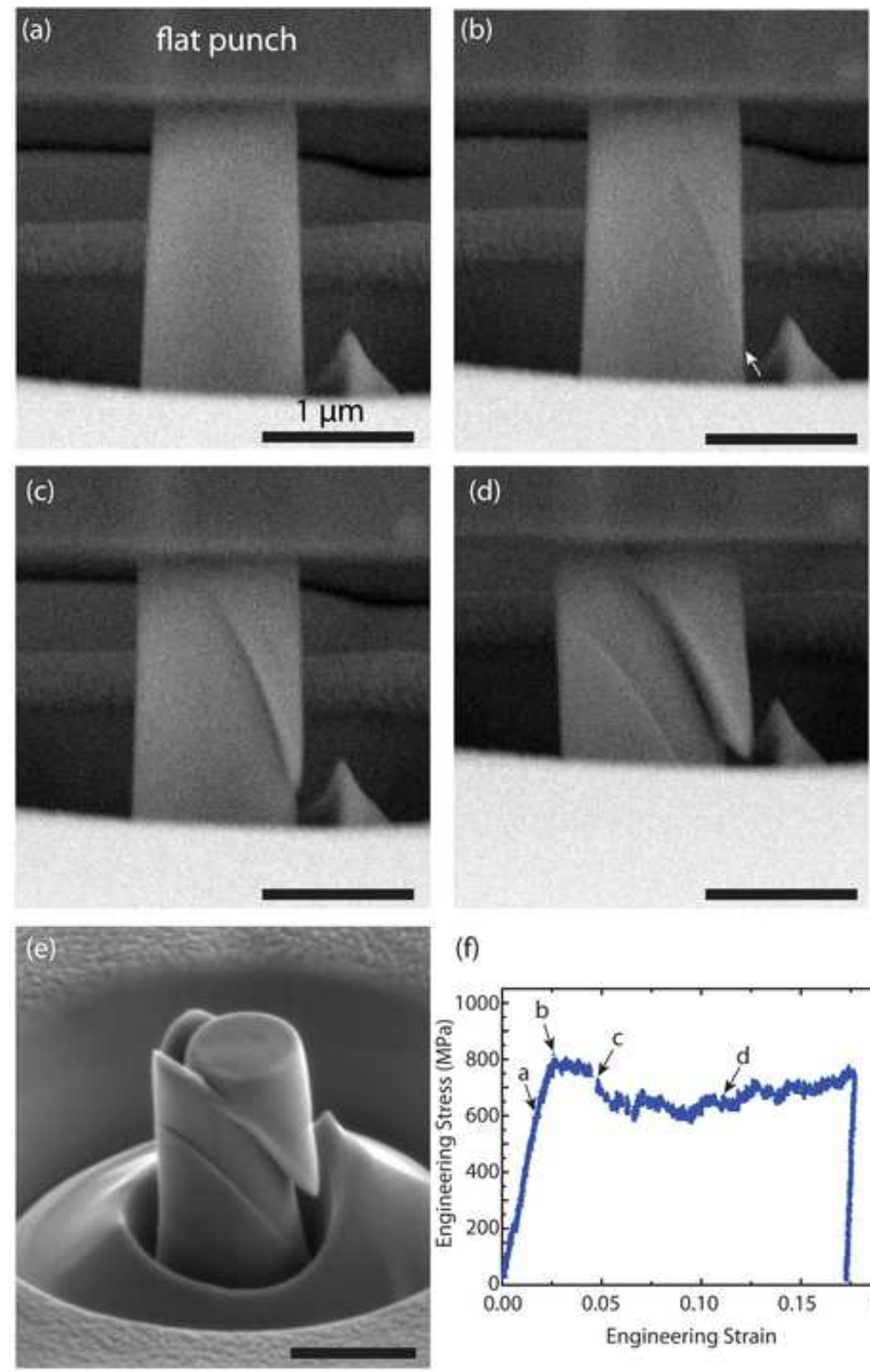

(f)

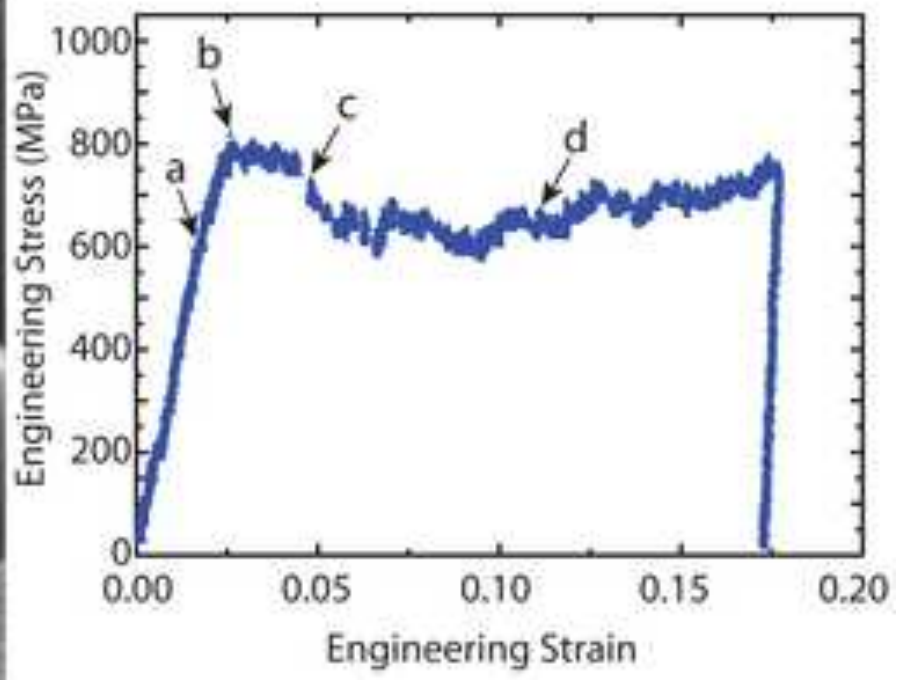




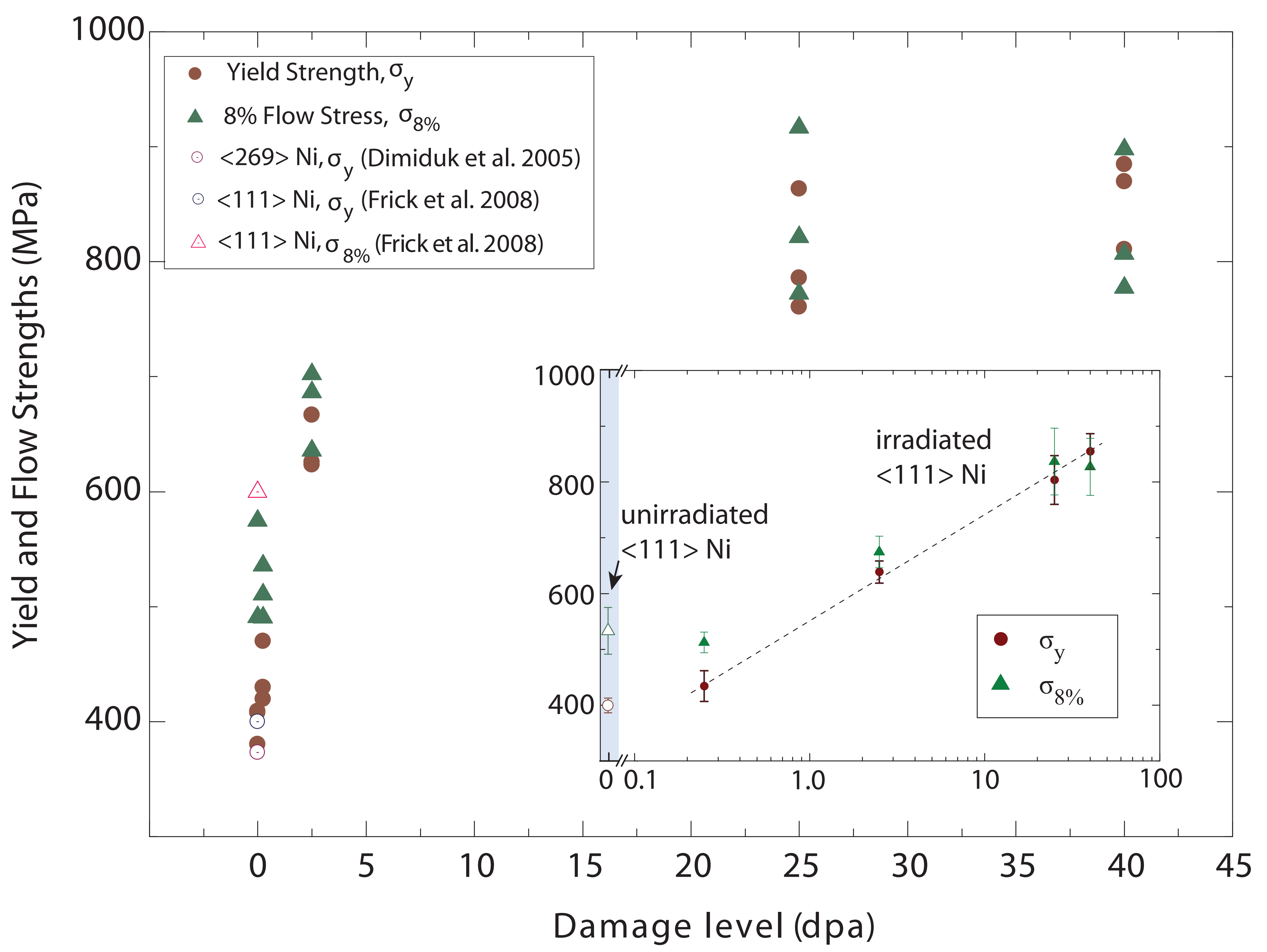


(a)

$0 \mathrm{dpa}$
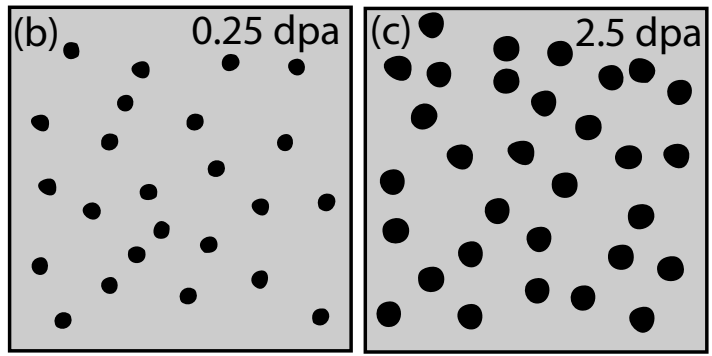

(d)

$25 \mathrm{dpa}$

(e) $-40 \mathrm{dpa}$

-

-

08

- 0 ○

$\bullet$

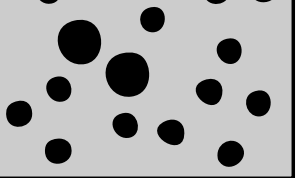


Tables and Captions

Table 1: Ion fluences, dose, and end of ranges in different irradiation region.

\begin{tabular}{c|c|c|c|c}
\hline & Region A & Region B & Region C & Region D \\
\hline $\begin{array}{c}\text { Fluence } \\
\left(\text { ions } / \mathrm{cm}^{2}\right)\end{array}$ & $6.31 \times 10^{16}$ & $3.71 \times 10^{16}$ & $3.71 \times 10^{15}$ & $3.71 \times 10^{14}$ \\
\hline $\begin{array}{c}\text { Mean damage } \\
\text { level, }<\beta> \\
(\mathrm{dpa})\end{array}$ & 40 & 25 & 2.5 & 0.25 \\
\hline $\begin{array}{c}\text { End of range } \\
(\mu \mathrm{m})\end{array}$ & $4.39 \pm 0.41$ \\
\hline
\end{tabular}

Table 2: Stress drop statistical scaling parameters including power law exponent $\tau$ and finite size cutoff $x_{0}$. The variance given in the values for $\tau$ and $x_{0}$ represent $95 \%$ confidence intervals for the fits.

\begin{tabular}{c|c|c|c}
\hline $\begin{array}{c}\text { Displacement } \\
\text { Damage (dpa) }\end{array}$ & $\boldsymbol{\tau}$ & $\boldsymbol{x}_{\mathbf{o}} \mathbf{( M P a )}$ & $\begin{array}{c}\text { Number of } \\
\text { Stress Drops }\end{array}$ \\
\hline \hline 0 & $1.51 \pm 0.02$ & $83.8 \pm 2.3$ & 105 \\
\hline 0.25 & $1.33 \pm 0.03$ & $16.6 \pm 0.2$ & 185 \\
\hline 2.5 & $1.81 \pm 0.03$ & $25.3 \pm 0.4$ & 118 \\
\hline 25 & $1.64 \pm 0.04$ & $29.2 \pm 1.2$ & 150 \\
\hline 40 & $1.58 \pm 0.02$ & $27.2 \pm 0.4$ & 199 \\
\hline
\end{tabular}

